\title{
Revisión sobre la evidencia de la relación entre exposición profesional al ruido y efectos extrauditivos no cardio-vasculares
}

\author{
Systematic review and evidence on occupational \\ Noise exposure and extra-auditory effects \\ of no cardiovascular nature
}

\author{
Jerónimo Maqueda Blasco \\ Escuela Nacional de Medicina del Trabajo. Instituto de Salud Carlos III. \\ Madrid. España.

\section{Rosana Cortés Barragán} \\ Escuela Nacional de Medicina del Trabajo. Instituto de Salud Carlos III. \\ Madrid. España.

\section{Elena Ordaz Castillo}

Escuela Nacional de Medicina del Trabajo. Instituto de Salud Carlos III. Madrid. España.

\section{Angel Asúnsolo del Barco}

Departamento de Ciencias Médico-Sociales. Universidad de Alcalá de Henares. Alcalá de Henares (Madrid). España

\section{Agustín Silva Mato}

Departamento de Ciencias Médico-Sociales. Universidad de Alcalá de Henares. Alcalá de Henares (Madrid). España

\section{Eva Bermejo García}

Escuela Nacional de Medicina del Trabajo. Instituto de Salud Carlos III. Madrid. España.

\section{Maria Fe Gamo González}

Escuela Nacional de Medicina del Trabajo. Instituto de Salud Carlos III. Madrid. España.

Recibido: 29-01-10

Aceptado: 18-02-10

\section{Correspondencia:}

Jerónimo Maqueda Blasco

Escuela Nacional de Medicina del Trabajo

Pabellón 8. Ciudad Universitaria

28040 Madrid. España

Tfno: 918224011

Fax: 915447073

E-mail: jmaqueda@isciii.es

Resumen

Trabajo de revisión que tienen como objetivo conocer el nivel de evidencia existente sobre los efectos extra-auditivos de la exposición profesional al ruido relacionados con: alteraciones bioquímicas, efectos neuro-piscológicos, salud reproductiva y accidentes de trabajo mediante el análisis sistemático de la producción científica publicada entre 1995 y 2008. La búsqueda de las referencias bibliográficas se realizó mediante términos "Major Topic" y "MeSH" para MEDLINE y términos "Decriptors" para OSH update. Se recuperaron un total de 370 referencias y se incluyeron en la revisión todos los estudios experimentales, estudios de cohortes y estudios casos control y aquellos estudios trasversales que cumplieron al menos con 
cinco de los nueve criterios que la declaración STROBE establece para los estudios observacionales. Fueron seleccionados para su revisión a texto completo un total de 35 artículos: 14 estudios experimentales, 2 estudios de cohortes, 6 estudios casos control, 12 estudios transversales y 1 estudio de validación.

La asignación del nivel de evidencia se realizó de acuerdo a los criterios SIGN. La selección y revisión de los artículos se realizó mediante revisión por pares, los casos de discrepancia entre pares se resolvió mediante consenso.

Encontramos un alto nivel de evidencia (1+) para influencia de la exposición profesional al ruido sobre el incremento de la secreción de cortisol y noradrenalina, fatiga y disminución del rendimiento, funciones cognitivas y memoria, disminución en la calidad del sueño, estrés e irritabilidad y percepción de malestar. Con un nivel de evidencia 2++ se relacionó la exposición profesional al ruido con la accidentadad laboral. No encontramos unos resultados concluyentes para la determinación de la relación entre exposición profesional al ruido sobre la salud reproductiva.

Existe una diversidad en los métodos utilizados para la evaluación de la exposición profesional al ruido, empleándose tanto métodos de medición cuantitativos y métodos cualitativos de percepción subjetiva.

Nuestros resultados son coincidentes con las revisiones realizadas anteriormente por Smith AP y cols (1991) y Butler MP y cols (1999).

Med Segur Trab (Internet) 2010; 56 (218): 49-71

Palabras clave: Ruido, salud laboral, salud reproductiva, accidentes de trabajo, efectos extra-auditivos, estrés, rendimiento, alteraciones del sueño.

Abstract

Review aimed at ascertaining the level of evidence on non-auditory effects related with occupational exposure to noise concerning: biochemical, neuro- psychology effects, reproductive health and accidents at work through a systematic analysis of the scientific literature published between 1995 and 2008. The search for references was conducted using terms "Major Topic" and "MeSH" terms for MEDLINE and "Decriptors" for $\mathrm{OSH}$ update. We retrieved a total of 370 references, were included in the review all experimental studies, cohort studies and case control studies and those cross-sectional

studies that had at least five of the nine criteria that the STROBE Statement provides for observational studies. Were selected for full text review a total of 35 studies: 14 experimental studies, 2 cohort studies, 6 case control studies, 12 cross-sectional studies and 1 validation study.

The assignment of the level of evidence was performed according to SIGN criteria. The selection and review of articles was done by peer review, cases of disagreement between pairs was resolved by consensus.

We found a high level of evidence (1+) about the influence of occupational noise exposure and the increased secretion of cortisol and norepinephrine, fatigue and decreased performance, cognitive function and memory, decreased quality of sleep, stress and irritability annoyance. A level of evidence 2++ was identify for occupational noise exposure and work accidents. We found no conclusive results in determine the relationship between occupational exposure to noise on reproductive health.

There is diversity in the methods used for assessing occupational exposure to noise, using both quantitative measurement methods and qualitative methods of subjective perception.

Our results are consistent with those previously reported by Smith AP et alt (1991) and Butler MP et al (1999).

Med Segur Trab (Internet) 2010; 56 (218): 49-71

Keywords: Noise, occupational health, reproductive bealth, work accidents, non auditory effects, stress, performance, sleep disturbances 


\section{INTRODUCCIÓN}

El ruido es uno de los agentes físicos más extendidos en el medio laboral. De acuerdo a los resultados de la Cuarta Encuesta Europea de Condiciones de Trabajo (1) el $30 \%$ de trabajadores europeos están expuestos a ruidos fuertes en su entorno de trabajo

En nuestro país la prevalencia de trabajadores que soportan un ruido molesto asciende a un 37\% según la VI Encuesta Nacional de Condiciones de Trabajo (VI ENCT) (2).

La legislación en materia de protección de los trabajadores orienta la acción preventiva a la vigilancia y control de los efectos auditivos derivados de la exposición laboral al ruido (3), sin embargo existen exposiciones laborales a niveles de presión sonora insuficiente para producir pérdidas auditivas o daños en el aparato auditivo pero que puede provocar efectos en otros órganos, interferir en la comunicación y en el desempeño de tareas (4).

Definimos los efectos extra-auditivos como todos aquellos efectos que afectan a la salud y al bienestar del sujeto y que son causados por exposición a ruido con exclusión de los efectos producidos directamente sobre el aparato auditivo o sobre la audición (5).

La Organización Mundial de la Salud (6) establece efectos cuantificables del ruido sobre el sueño a partir de $30 \mathrm{~dB}(\mathrm{~A})$, interferencias en la comunicación oral por encima de los $35 \mathrm{~dB}$ (A); perturbaciones en el individuo a partir de los $50 \mathrm{~dB}$ (A); efectos cardiovasculares por exposición a largo plazo al ruido de $65-70 \mathrm{~dB}(\mathrm{~A})$ y una reducción de la actitud cooperativa y un aumento en el comportamiento agresivo en individuos predispuestos a la agresividad por encima de $80 \mathrm{~dB}(\mathrm{~A})$, así como una relación entre exposición a ruido y alteraciones hormonales que conlleva a un desequilibrio en el sistema endocrino e inmune.

En general, estos efectos extra-auditivos están mediados por una reacción de estrés a la contaminación acústica (7), desencadenando en el organismo una respuesta a los estímulos auditivos como lo haría ante cualquier agresión de tipo físico o psíquico.

Hay autores que (8) observan una activación del eje hipotálamo hipófiso adrenal (HHA) producido por la exposición a ruido de forma prolongada, que lleva a aumentar periódicamente y de forma anómala los niveles de cortisol produciendo un número de efectos que desequilibran la balanza hormonal y pueden causar alteraciones de tipo respiratorio, con aumento de la frecuencia respiratoria (9), alteraciones digestivas, con aumento de la acidez gástrica e incremento de la incidencia de las úlceras gastroduodenales (10) y alteraciones cardiovasculares (11).

En este sentido, la OMS se encuentra actualmente realizando un estudio piloto sobre morbilidad y mortalidad cardiovascular atribuible al ruido dentro del programa indicadores de ambiente y salud (12). Este programa contempla, entre otros efectos extra-auditivos el malestar y las alteraciones del sueño debido al ruido.

Diversos estudios evidencian que una exposición a un nivel de ruido de $45 \mathrm{~dB}$ produce un incremento en el periodo de latencia del sueño, disminuyendo sus fases profundas necesarias para un sueño reparador y originando un estado de cansancio crónico en los individuos expuestos (13).

Como resultado se produce una mala calidad del sueño que puede afectar al ámbito laboral disminuyendo la capacidad para el trabajo, alterando las funciones diurnas, el bienestar del individuo y aumentando la morbilidad con síntomas de cansancio, irritabilidad, aumento de la agresividad, alteraciones del rendimiento, ansiedad, angustia, labilidad emocional, apatía, irritabilidad, alteraciones del apetito, etc

Bajos niveles de ruido producen además malestar, entendido éste como un sentimiento de rechazo hacia el agente estresante (ruido), que se traduce en una serie reacciones conductuales tales como irritabilidad, labilidad emocional, ansiedad o angustia que, aunque normalmente son puntuales, pueden cronificarse y constituirse en enfermedad (depresión, conductas paranoides, etc) (14). 
Por otro lado, distintos autores analizan los probables efectos de la exposición a ruido en la gestación con alteraciones prenatales como el bajo peso neonatal (15), la prematuridad (16-18), abortos u otros efectos de la reproducción. Existen diversas teorías sobre los posibles mecanismos de actuación del ruido en el feto que contemplan desde una disminución del flujo útero -placentario resultando en hipoxia fetal (19) y aumento de la secreción materna de catecolaminas (18).

Otros autores sugieren que el ambiente intrauterino permite el paso de sonidos externos hacia del útero fácilmente o que existe un efecto indirecto del ruido transmitido, produciendo una respuesta nerviosa inducida que puede generar cambios en la presión arterial y aumentos de flujo cardiovascular.

En relación a la mayor accidentabilidad laboral, grupos de trabajo de la década de los 70 afirmaban que los trabajadores profesionalmente expuestos a ruido tenían un riesgo 3 veces superior de sufrir accidentes de trabajo (20). Más recientemente otros estudios han verificado esta asociación entre una exposición laboral a ruido (alrededor de $82 \mathrm{dBA}$ ), y la probabilidad de sufrir accidentes de trabajo (21).

El ruido, por tanto, puede provocar malestar, disminuir o impedir la atención, alterar la capacidad de concentración, el sueño, el rendimiento, inducir comportamientos psicológicos alterados, causar accidentes de trabajo, provocar alteraciones fisiológicas en el sistema cardiovascular e inducir posibles alteraciones fetales etc. (22).

Los indicadores de prevalencia, en base a los datos de encuestas de condiciones de trabajo, ponen de manifiesto que, por su extensión, el control de la exposición laboral al ruido sigue siendo un problema actual en el ámbito de la mejora de la calidad de vida y condiciones de trabajo.

En este trabajo se realiza una revisión sistemática de la literatura sobre efectos extraauditivos no cardiovasculares por exposición laboral al ruido en población trabajadora publicada entre 1995 -2008 analizando efectos sobre las catecolaminas, efectos psicosociales (incluyendo malestar, sueño, rendimiento y otros), alteraciones fetales y accidentes de trabajo.

Se ha tomado como referencia dos trabajos de revisión previos sobre los efectos extra-auditivos del ruido y publicados por la HSE $(5,23)$ con el objeto de encontrar las posibles diferencias, similitudes o nuevos hallazgos de la literatura publicada desde la fecha de la última revisión en 1995 hasta el año 2008 y ver si ha mejorado la evidencia en estos últimos 14 años.

Se añade a nuestra revisión, un análisis de la evidencia científica mediante la aplicación de los niveles de evidencia científica, según el Scottish Intercollegiate Guidelines Network (SIGN) (24).

\section{OBJETIVOS}

Esta revisión se dirige a la consecución de dos objetivos:

1. Resumir el estado actual (1995-2008) del Conocimiento y sus niveles de Evidencia Científcia sobre exposición laboral al ruido y efectos extraauditivos-no cardiovasculares- tales como secreción de catecolaminas, alteraciones psicosociales (incluyendo malestar, sueño, rendimiento y otros), trastornos fetales y accidentes de trabajo.

2. Caracterizar la producción científica según diseño de los trabajos publicados y calidad metodológica.

\section{MATERIAL Y METODOS}

La identificación de la producción científica publicada entre 1995 y 2008 en relación con los efectos extra-auditivos no cardio-vasculares, en población trabajadora laboralmente 
expuesta a rudio se realizó a través de una estrategia de búsqueda con términos "Mesh y major topics” para Medline y términos "Descriptors” para OSH (tabla 1).

Tabla 1: Estrategia de búsqueda en MEDLINE y OSH UPDATE

Noise AND (Stress OR stress audit)

Noise AND Biochemical effects

Catecholamines AND noise

Catecholamines AND Occupational noise] Limits: (**)

((“Catecholamines"[Mesh] OR "Hydrocortisone"[Mesh]) OR “Cortisone”[Mesh]) AND Occupational noise (***)

Noise AND Performance

("Employee Performance Appraisal" OR Accidents OR Occupational accidents) AND Occupational noise] (**)

Occupational noise AND ((“Employee Performance Appraisal”[Mesh] OR (“Accidents"[Mesh] OR "Accidents, Occupational"[Mesh])) (*)

Noise AND ("PSYCHOSOCIAL EFFECTS" OR PSYCHOSOCIAL OR "PSYCHOLOGICAL DISORDERS" OR "PSYCHOLOGICAL EFFECTS")

("Personal Satisfaction"[Mesh]) ANd Occupational noise

Noise AND (Sleep disorders OR sleep OR sleep deprivation OR sleep disturbances)

(“Sleep Deprivation”[Mesh] OR “Sleep Disorders”[Mesh]) And Occupational Noise. ] (**)

Noise AND (Fatigue OR annoyance) AND Year of Publication 1995-2008

("Stress, Physiological"[Mesh] OR "Fatigue"[Mesh]) OR "Psychology"[Mesh]) AND Occupational noise [Mesh] ] Limits: Publication Date from 1999/01/01 to 2008/12/31) (**)

(("Stress, Physiological”[Mesh] OR "Fatigue"[Mesh]) OR "Psychology"[Mesh]) AND occupational noise (*)

Noise AND Year of Publication 1999-2008 AND (ANXIETY OR DEPRESSION OR IRRITATION

Occupational noise AND (((“Anxiety”[Mesh] OR “Depression”[Mesh]) OR “Memory”[Mesh]) OR

"Attention"[Mesh] (**)

Occupational noise AND (((“Anxiety”[Mesh] OR “Depression”[Mesh]) OR “Memory”[Mesh]) OR

"Attention"[Mesh]) (*)

Occupational noise AND ("Irritable Mood"[Mesh] OR ("Attention Deficit Disorder with Hyperactivity"[Mesh] OR "Borderline Personality Disorder"[Mesh]) ] (**)

Occupational noise AND ((((c"Sleep Deprivation"[Mesh] OR “Sleep Disorders”[Mesh])) OR “Irritable Mood”[Mesh]) OR “Attention Deficit Disorder with Hyperactivity”[Mesh]) OR “Borderline Personality Disorder”[Mesh]) (*)

Noise AND Accidents

Noise AND Accidents AND Year of Publication 1995-2008

Noise AND Year of Publication 1995-2008 AND (REPRODUCTION OR "REPRODUCTION DISORDERS" OR "REPRODUCTION DISORDER")

Noise AND Teratogenic effects

Noise AND Year of Publication 1995-2008 AND (FETAL OR "FETAL LOSS" OR "FETAL MALFORMATIONS" OR PREGNANCY OR DELIVERY OR BIRTH)

Occupational noise AND (((("Reproduction”[Mesh] OR "Fetal Development”[Mesh]) OR "Pregnancy”[Mesh]) OR “Delivery, Obstetric”[Mesh]) OR (“Term Birth”[Mesh] OR "Premature Birth”[Mesh]) (**)

Occupational noise AND (“Fetal Death"[Mesh] OR "Fetal Development”[Mesh]) (*)

((((“Reproduction”[Mesh] OR "Fetal Development”[Mesh]) OR “Pregnancy”[Mesh]) OR “Delivery,

Obstetric"[Mesh]) OR ("Term Birth"[Mesh] OR "Premature Birth"[Mesh])) AND Occupational noise (*)

"Respiratory system “ AND Occupational noise 
Para su revisión a texto completo se incluyeron los artículos que cumplían los siguientes criterios:

1. Año de publicación entre 1995 y 2008.

2. El estudio debía de cuantificar, medir o describir la relación entre el ruido y el efecto extra-auditivo analizado.

3. Ensayos, estudios experimentales, metanálisis, estudios de cohorte y estudios casos control.

4. Estudios observacionales transversales que cumplieran los requisitos de calidad metodológica de la Iniciativa STROBE.

5. Estudios publicados en ingles o español.

La calidad de los estudios transversales se evaluó según la declaración de la Iniciativa STROBE para la comunicación de estudios observacionales. Se incluyeron en la revisión aquellos artículos que cumplían 5 ó más puntos esenciales de los 9 criterios que para el apartado método describe esta declaración (25)

La revisión sistemática de los artículos seleccionados incluyó: el análisis de la publicación siguiendo los criterios de Sackett et al y Jadad (26-31), y la información en relación a las variables relacionadas en la tabla 2 .

Tabla 2: Aspectos del análisis sistemático de los artículos

\section{CARACTERÍSTICAS ESTUDIADAS}

Tipo de estudio

Población de estudio

Variable de exposición o ruido,

Variable a estudio analizada

Factores de confusión y técnicas estadísticas usadas

Resultados del estudio

Nivel de evidencia

A cada uno de los efectos estudiados en los artículos revisados se les asignó un nivel de evidencia científica de acuerdo a los criterios del Scottish Intercollegiate Guidelines Network (SIGN), (tabla 3). 
Tabla 3: Criterios de Evidencia científica y grados de recomendación (SIGN)

\begin{tabular}{|c|c|c|c|}
\hline $\begin{array}{l}\text { Grado de } \\
\text { evidencia }\end{array}$ & Criterios de evidencia & Criterios para recomendación & $\begin{array}{l}\text { Grado de } \\
\text { recomendación }\end{array}$ \\
\hline $1++$ & $\begin{array}{l}\text { Metanálisis, revisiones } \\
\text { sistemáticas de estudios } \\
\text { aleatorizados y controlados } \\
\text { (EAC) o EAC de alta calidad } \\
\text { y con muy bajo riesgo de } \\
\text { sesgo }\end{array}$ & $\begin{array}{l}\text { Al menos un metanálisis, una revisión } \\
\text { sistemática o un EAC clasificado como 1++ } \\
\text { y directamente aplicable a la población } \\
\text { diana de la GPC, o una revisión } \\
\text { sistemática de EAC o un conjunto de } \\
\text { evidencia constituido principalmente por } \\
\text { estudios clasificados como 1+ } \\
\text { directamente aplicables a la población } \\
\text { diana de la GPC y que muestran } \\
\text { coherencia en sus resultados }\end{array}$ & A \\
\hline $1+$ & $\begin{array}{l}\text { Metanálisis, revisiones } \\
\text { sistemáticas de EAC o EAC } \\
\text { bien desarrollados y con } \\
\text { bajo riesgo de sesgo. } \\
\text { Metanálisis, revisiones } \\
\text { sistemáticas de EAC o EAC } \\
\text { con alto riesgo de sesgo. }\end{array}$ & $\begin{array}{l}\text { Un conjunto de evidencia constituido por } \\
\text { estudios clasificados como } 2++ \\
\text { directamente aplicables a la población } \\
\text { diana de la GPC y que muestran } \\
\text { coherencia en sus resultados, o evidencia } \\
\text { extrapolada a partir de estudios } \\
\text { clasificados como } 1++ \text { o } 1+\text {. }\end{array}$ & B \\
\hline $2++$ & $\begin{array}{l}\text { Revisiones sistemáticas de } \\
\text { estudios de casos y } \\
\text { controles o de estudios de } \\
\text { cohortes de alta calidad o } \\
\text { estudios de casos y } \\
\text { controles, y estudios de } \\
\text { cohortes con muy bajo } \\
\text { riesgo de sesgo y con } \\
\text { elevada probabilidad de } \\
\text { que la relación sea causal. } \\
\text { Estudios de casos y } \\
\text { controles y estudios de } \\
\text { cohortes bien } \\
\text { desarrollados, con bajo } \\
\text { riesgo de sesgo y con } \\
\text { probabilidad moderada de } \\
\text { que la relación sea causal. }\end{array}$ & $\begin{array}{l}\text { Un conjunto de evidencia constituido por } \\
\text { estudios clasificados como } 2+ \\
\text { directamente aplicables a la población } \\
\text { diana de la GPC y que muestran } \\
\text { coherencia en sus resultados, o evidencia } \\
\text { extrapolada a partir de estudios } \\
\text { clasificados como } 2++.\end{array}$ & $\mathrm{C}$ \\
\hline $2-$ & $\begin{array}{l}\text { Estudios de casos y } \\
\text { controles, y estudios de } \\
\text { cohortes con alto riesgo de } \\
\text { sesgo y con riesgo } \\
\text { importante de que la } \\
\text { relación no sea causal. }\end{array}$ & & \\
\hline 3 & $\begin{array}{l}\text { Estudios no analíticos, } \\
\text { como informes de casos y } \\
\text { series de casos }\end{array}$ & $\begin{array}{l}\text { Evidencia de nivel } 3 \text { o } 4 \text {, o evidencia } \\
\text { extrapolada desde estudios clasificados } \\
\text { como } 2+.\end{array}$ & $\mathrm{D}$ \\
\hline 4 & Opinión de expertos & $\begin{array}{l}\text { Puntos de Buena Praxis: mejor práctica } \\
\text { recomendada basada, por consenso, en la } \\
\text { experiencia clínica de los miembros de los } \\
\text { grupos que han desarrollado la GPC. }\end{array}$ & $\mathrm{BP}$ \\
\hline
\end{tabular}


La identificación y revisión de los artículos se realizó en tres fases diferentes:

En una primera fase dos documentalistas expertos en documentación biomédica, reprodujeron, de forma independiente la estrategia de búsqueda.

En una segunda etapa dos revisores del ámbito de la medicina del trabajo verificaron las publicaciones identificadas y realizaron una primera selección de las mismas de acuerdo a los criterios de selección.

En una tercera fase un equipo multidisciplinar de cuatro investigadores (del ámbito de la medicina del trabajo, epidemiología y metodología de investigación) mediante revisión por pares, cribaron los estudios al objeto de consolidar la selección realizada.

En caso de discrepancia entre pares se llegó a un acuerdo mediante consenso.

\section{RESULTADOS}

En total se recuperaron un total de 370 referencias de artículos publicados entre 1995 y 2008. Tras aplicar los filtros descritos previamente y los criterios de inclusión y exclusión, así como los criterios de calidad de estudios observacionales de la declaración Strobe para se seleccionaron un total de 35 artículos para revisión a texto completo, cuya distribución por tipo de estudio y efecto estudiado figura en la tabla 4.

Tabla 4. Distribución del número de artículos de revisión a texto completo según efecto estudiado y tipo de diseño del estudio

\begin{tabular}{lccccc}
\hline Efecto & Experimental & Casos-Control & Cohorte & Transversal & TOTAL \\
\hline $\begin{array}{l}\text { Efectos Bioquímicos y } \\
\text { Catecolaminas }\end{array}$ & $6\left(1^{*}\right)$ & & & $2\left(1^{*}\right)$ & 8 \\
Salud reproductiva & & 3 & 1 & 2 & 6 \\
Neuro- psicológico & $8\left(3^{*}\right)$ & 3 & 1 & $8\left(1^{*}\right)$ & $17\left(4^{*}\right)$ \\
Accidentes & & 6 & 2 & 1 & 4 \\
Total & 14 & & & 13 & 35 \\
\hline
\end{tabular}

(*) número de estudios analizados por estudiar más de un efecto

Por tipo de efecto analizado fueron más frecuentes los estudios relacionados con los efectos neuro-psciológicos (17 estudios) y por diseño empleado predominaron los estudios experimentales (14 estudios) seguido de los estudios con diseño de corte o transversales (13 estudios).

Efecto de la exposición profesional a ruido sobre efectos bioquímicos y/o catecolaminas (tabla 5) 
Tabla 5. Publicaciones analizadas sobre exposición laboral a ruido y efectos bioquímicos y catecolaminas

\begin{tabular}{|c|c|c|c|c|c|c|c|}
\hline $\begin{array}{c}\text { PRIMER } \\
\text { AUTOR } \\
\text { AÑN/ (REF) }\end{array}$ & $\begin{array}{l}\text { TIPO DE } \\
\text { DISEÑO }\end{array}$ & $\mathbf{N}$ & $\begin{array}{l}\text { UMBRAL } \\
\text { DE RUIDO } \\
\text { dBA }\end{array}$ & $\begin{array}{c}\text { VARIABLE } \\
\text { RESULTADO }\end{array}$ & RESULTADOS & $\begin{array}{l}\text { F.C. } \\
\text { (1) }\end{array}$ & $\begin{array}{l}\text { N.E. } \\
\text { (2) }\end{array}$ \\
\hline $\begin{array}{l}\text { Melamed, S } \\
1996(32)\end{array}$ & Experimental & 35 & $>85$ & $\begin{array}{l}\text { Alteración } \\
\text { cortisol } \\
\text { urinario(*) }\end{array}$ & $\begin{array}{l}\text { Disminución de los } \\
\text { Niveles de cortisol tras } \\
\text { condiciones de } \\
\text { reducción de ruido } \\
(\mathrm{p}<0,05) \text { con uso de } \\
\text { EPIs }\end{array}$ & + & $1+$ \\
\hline $\begin{array}{l}\text { Miki K. } \\
1998 \text { (33) }\end{array}$ & Experimental & 9 & 90 & $\begin{array}{l}\text { Aumento de } \\
\text { cortisol salivar } \\
\text { y urinario, } \\
\text { catecolaminas } \\
\text { en orina }(*)\end{array}$ & $\begin{array}{l}\text { No diferencias } \\
\text { significativas en la } \\
\text { secreción de } \\
\text { adrenalina. } \\
\text { Aumento de cortisol } \\
\text { urinario (p<0,001). } \\
\text { Aumento de cortisol } \\
\text { salivar }(\mathrm{p}<0,05)\end{array}$ & + & $1+$ \\
\hline $\begin{array}{l}\text { Mocci F. } \\
2001(34)\end{array}$ & Experimental & 95 & $98-108$ & $\begin{array}{l}\text { Sangre: } \\
\text { Magnesio, } \\
\text { calcio, } \\
\text { fósforo., } \\
\text { catecolaminas } \\
\text { Orina: } \\
\text { Magnesio, } \\
\text { calcio, fosfatos } \\
\text { y creatinina }\end{array}$ & $\begin{array}{l}\text { Incremento del Calcio } \\
\text { y Magnesio séricos } \\
\text { No diferencias } \\
\text { significativas de } \\
\text { catecolaminas y } \\
\text { fósforo. } \\
\text { Incremento } \\
\text { significativo en } \\
\text { secreción urinaria de: } \\
\text { magnesio y fosfatos } \\
\text { No diferencias } \\
\text { significativas en } \\
\text { secreción urinaria de: } \\
\text { Calcio, creatinina }\end{array}$ & + & $1+$ \\
\hline $\begin{array}{l}\text { Miyakawa, } \\
\text { M. } 2006 \text { (35) }\end{array}$ & Experimental & 20 & 90 & $\begin{array}{l}\text { Aumento de } \\
\text { CgA salivar }\end{array}$ & $\begin{array}{l}\text { Aumento de los } \\
\text { niveles de Cg A salivar } \\
\text { durante la exposición } \\
\text { al ruido (test de } \\
\text { Friedman } p=0,001 \text {, dos } \\
\text { ramas }+ \text { ) }\end{array}$ & + & $1+$ \\
\hline $\begin{array}{l}\text { Gitanjali B, } \\
2003(36)(*)\end{array}$ & $\begin{array}{l}\text { Estudio } \\
\text { experimental } \\
\text { prospectivo, } \\
\text { cruzado. }\end{array}$ & 10 & $>75(8 \mathrm{~h})$ & $\begin{array}{l}\text { Cortisol en } \\
\text { sangre }\end{array}$ & $\begin{array}{l}\text { Incremento de cortisol } \\
\text { en sangre tras } \\
\text { exposición diurna a } \\
\text { ruido }(\mathrm{p}<0,005)\end{array}$ & - & $1+$ \\
\hline $\begin{array}{l}\text { Ising H. } \\
2004(37)(*)\end{array}$ & Experimental & 42 & 60 y 97 & $\begin{array}{l}\text { Excrecion de } \\
\text { catecolaminas } \\
\text { (adrenalina, } \\
\text { noradrenalina } \\
\text { y Camp } \\
\text { en orina) (*) }\end{array}$ & $\begin{array}{l}\text { Incremento } \\
\text { significativo de } \\
\text { noradrenalina en } \\
(>10 \% \text { en } 41 \text { sujetos) }\end{array}$ & + & $1+$ \\
\hline $\begin{array}{l}\text { Sudo, A } \\
1996(38)\end{array}$ & Transversal & 75 & $93-100$ & $\begin{array}{l}\text { Catecolaminas } \\
\text { y cortisol en } \\
\text { orina }\end{array}$ & $\begin{array}{l}\text { Coeficiente de } \\
\text { correlación entre la } \\
\text { frecuencia de pulso y } \\
\text { la excreción de } \\
\text { noradrenalina }(\mathrm{p}<0,01) \\
\text { y adrenalina }(\mathrm{p}<0,05) \\
\text { La excreción de } \\
\text { catecolaminas } \\
\text { disminuyen con el uso } \\
\text { de EPI }\end{array}$ & + & 3 \\
\hline $\begin{array}{l}\text { Rojas- } \\
\text { González L, } \\
2004 \text { (39) }\end{array}$ & Transversal & 40 & $>85$ & Cortisol sérico & $\begin{array}{l}\text { Incremento de los } \\
\text { valores de cortisol } \\
\text { sérico después de la } \\
\text { jornada }(\mathrm{p}<0,05) .\end{array}$ & - & 3 \\
\hline
\end{tabular}

(1) Control de posibles factores de confusión

(2) Nivel de Evidencia

(*)Articulo analizado dos veces por estudiar distintos efectos 
La tabla 5 resume los ocho artículos revisados (6 de diseño experimental y dos transversales) relativos a la influencia de la exposición laboral a ruido sobre la secreción de catecolaminas, cortisol y algunos electrolitos como magnesio, calcio y fósforo.

Bajo umbrales de exposición a ruido que oscilan en estos estudios entre 60 y 108 dBA, los efectos más frecuentemente analizados fueron los cambios en el cortisol (5 estudios), catecolaminas ( 4 estudios), electrolitos ( 1 estudio) y cambios inmunológicos ( 1 estudio).

Los resultados tanto de los estudios experimentales como transversales evidencian una asociación entre exposición profesional a ruido e incremento de los niveles de cortisol con independencia del medio en que se ha analizado (sérico, urinario o salivar). La evidencia para este efecto es de $1+$.

El incremento de los niveles de noradrenalina en trabajadores expuestos a ruido se pone de manifiesto en 1996 en el estudio de diseño transversal de Sudo A. (38). Este hallazgo coincide con el resultado del estudio experimental realizado por Ising $\mathrm{H}$ (37) en 2004. El nivel de evidencia para el incremento de noradrenalina es de 1+.

En cuanto a los niveles de catecolaminas, los resultados de los estudios experimentales coinciden en no evidenciar una asociación entre exposición profesional a ruido y los niveles de concentración para otras catecolaminas (nivel de evidencia 1+).

La influencia de la exposición a ruido sobre algunos electrolitos es analizada en el estudio experimental de Mocci F (34) evidenciándose una asociación entre exposición a ruido y niveles de calcio y magnesio séricos y de magnesio y fosfatos en orina (nivel de evidencia 1+).

Por otro lado, Miyakawa M (36) en un estudio experimental, evidencia una relación entre exposición a ruido e incremento en la cromogranina A salivar (nivel de evidencia 1+).

Efectos neuro-psicológicos por exposición profesional a ruido (tabla 6)

Tabla 6: Publicaciones analizadas sobre exposición laboral a ruido y efectos neuro-psicológicos

\begin{tabular}{|c|c|c|c|c|c|c|c|}
\hline $\begin{array}{c}\text { PRIMER } \\
\text { AUTOR } \\
\text { AÑO/ (REF) }\end{array}$ & $\begin{array}{l}\text { TIPO DE } \\
\text { DISEÑO }\end{array}$ & $\mathbf{N}$ & $\begin{array}{l}\text { UMBRAL } \\
\text { DE } \\
\text { RUIDO } \\
\text { dBA }\end{array}$ & $\begin{array}{l}\text { VARIABLE } \\
\text { RESULTADO }\end{array}$ & RESULTADOS & F.C(1) & $\begin{array}{l}\text { N.E. } \\
\text { (2) }\end{array}$ \\
\hline $\begin{array}{l}\text { Murthy V.S. } \\
1995 \text { (40) }\end{array}$ & $\begin{array}{l}\text { Cuasi } \\
\text { experimental }\end{array}$ & 20 & 77.32 & $\begin{array}{l}\text { Funciones } \\
\text { cognitivas: } \\
\text { Eficiencia mental y } \\
\text { memoria a corto } \\
\text { plazo. }\end{array}$ & $\begin{array}{l}\text { El ruido, reduce la } \\
\text { eficiencia mental y la } \\
\text { memoria a corto plazo. } \\
\text { La disminución de la } \\
\text { puntuación en los test } \\
\text { antes y después de la } \\
\text { intervención con ruido } \\
\text { es estadísticamente } \\
\text { significativo }(p<0,05) \text {. }\end{array}$ & + & $1-$ \\
\hline $\begin{array}{l}\text { Gitanjali B, } \\
2003(36) \\
(*)\end{array}$ & Experimental & 10 & $>75(8 \mathrm{~h})$ & $\begin{array}{l}\text { Cambios } \\
\text { en el Sueño } \\
\text { Nocturno, Cambios } \\
\text { en frecuencia } \\
\text { cardiaca } \\
\text { y cortisol realizado } \\
\text { durante } \\
4 \text { noches sucesivas }\end{array}$ & $\begin{array}{l}\text { La eficiencia de sueño fue } \\
\text { menos del } 80 \% \\
\text { y el tiempo total pasado } \\
\text { en sueño REM, onda } \\
\text { corta, latencia de REM fue } \\
\text { significativamente menor } \\
\text { en la noche después de la } \\
\text { exposición a ruido. }\end{array}$ & + & 1- \\
\hline $\begin{array}{l}\text { Melamed, S } \\
1996 \text { (41) }\end{array}$ & Experimental & 35 & $>85$ & $\begin{array}{l}\text { Irritabilidad post } \\
\text { laboral }(*) \\
\text { rendimiento } \\
\text { y fatiga }\end{array}$ & $\begin{array}{l}\text { Reducción de fatiga, } \\
(\mathrm{p}<0.05) \text { y de irritabilidad } \\
\text { postlaboral }(\mathrm{p}<0.01) \text {. } \\
\text { Contribución neta del } \\
\text { ruido para elevar el estrés } \\
\text { laboral en demandas } \\
\text { regulares de trabajo }\end{array}$ & + & $1+$ \\
\hline $\begin{array}{l}\text { Miki K, \& } \\
1998(42)\end{array}$ & Experimental & 9 & 90 & $\begin{array}{l}\text { Irritabilidad } \\
\text { estres(*) }\end{array}$ & $\begin{array}{l}\text { Los sujetos se sienten } \\
\text { más irritables cuando } \\
\text { realizan las tareas con } \\
\text { ruido( } 2.25+/-0.65) \text { que } \\
\text { bajo condiciones } \\
\text { tranquilas } \\
\left(1+/ \_0,27\right) ; \mathrm{p}<0,05\end{array}$ & + & $1+$ \\
\hline
\end{tabular}




\begin{tabular}{|c|c|c|c|c|c|c|c|}
\hline $\begin{array}{c}\text { PRIMER } \\
\text { AUTOR } \\
\text { AÑNO/ (REF) }\end{array}$ & $\begin{array}{l}\text { TIPO DE } \\
\text { DISEÑO }\end{array}$ & $\mathbf{N}$ & $\begin{array}{l}\text { UMBRAL } \\
\text { DE } \\
\text { RUIDO } \\
\text { dBA }\end{array}$ & $\begin{array}{l}\text { VARIABLE } \\
\text { RESULTADO }\end{array}$ & RESULTADOS & F.C(1) & $\begin{array}{l}\text { N.E. } \\
(2)\end{array}$ \\
\hline $\begin{array}{l}\text { Persson,W } \\
2001 \text { (43) }\end{array}$ & Experimental & 32 & 40 & $\begin{array}{l}\text { Rendimiento y } \\
\text { malestar, humor, } \\
\text { estres, esfuerzo } \\
\text { molestias } \\
\text { y presencia } \\
\text { de síntomas }\end{array}$ & $\begin{array}{l}\text { El ruido de baja } \\
\text { frecuencia es percibido } \\
\text { como más molesto y } \\
\text { repercute más sobre la } \\
\text { capacidad de trabajo } \\
\text { Los sujetos clasificados } \\
\text { como más sensibles a } \\
\text { altas frecuencias de ruido } \\
\text { son los que tienen mayor } \\
\text { riesgo }\end{array}$ & + & $1+$ \\
\hline $\begin{array}{l}\text { Ising, H. } 2004 \\
\text { (37) } \\
(*)\end{array}$ & Experimental & 42 & $60-97$ & $\begin{array}{l}\text { Estrés, rendimiento } \\
\text { y fatiga }\end{array}$ & $\begin{array}{l}\text { Encontraron cambios } \\
\text { psicológicos y por estrés } \\
\text { y alteraciones fisiológicas } \\
\text { debido a ruido, en la } \\
\text { mitad de los sujetos } \\
\text { estudiados; el aumento } \\
\text { de tensión o fatiga } \\
\text { mental se correlacionó } \\
\text { tanto con ascensos, como } \\
\text { con descensos de la } \\
\text { tensión arterial }\end{array}$ & + & $1+$ \\
\hline $\begin{array}{l}\text { Muzammil, M. } \\
2004 \text { (44) }\end{array}$ & Experimental & 5 & $\begin{array}{l}80,90 \\
\text { y } 100\end{array}$ & Rendimiento & $\begin{array}{l}\text { El nivel de ruido y carga } \\
\text { tenían ambos efectos } \\
\text { significativos en el } \\
\text { rendimiento. Pero la } \\
\text { interacción entre ambos } \\
\text { no fue significativa }\end{array}$ & + & $1+$ \\
\hline $\begin{array}{l}\text { Pawlaczyk- } \\
\text { Luszczyniska } \\
\text { M. } 2005 \text { (45) }\end{array}$ & Experimental & 96 & 50 & $\begin{array}{l}\text { Rendimiento } \\
\text { (funciones visuales, } \\
\text { concentración } \\
\text { y atención selectiva } \\
\text { y continua) } \\
\text { y bienestar } \\
\text { subjetivo } \\
4 \text { test psicológicos } \\
\text { estandarizados }\end{array}$ & $\begin{array}{l}\text { Exposición a bajas } \\
\text { frecuencia a niveles } \\
\text { moderados, pueden } \\
\text { afectar negativamente a } \\
\text { las funciones visuales, } \\
\text { concentración y atención } \\
\text { selectiva, especialmente } \\
\text { en sujetos de alta } \\
\text { sensibilidad a baja } \\
\text { frecuencias }\end{array}$ & + & $1+$ \\
\hline $\begin{array}{l}\text { Gitanjali B, } \\
2003(46)\end{array}$ & Cohorte & 24 & $>75$ & $\begin{array}{l}\text { Alteraciones } \\
\text { del Sueño }\end{array}$ & $\begin{array}{l}\text { Fuerte asociación entre } \\
\text { exposición ocupacional a } \\
\text { alto ruido y pobre } \\
\text { eficiencia de sueño (RR } \\
2.49 ; \text { I.C al } 95 \% 1.12 \text { - } \\
5.57 ; \mathrm{P}=0.01 .\end{array}$ & + & $2++$ \\
\hline $\begin{array}{l}\text { Rios AL, } 2005 \\
\text { (47) }\end{array}$ & Transversal & 40 & $>85$ & $\begin{array}{l}\text { Calidad } \\
\text { del sueño con } \\
\text { polisomnografia } \\
\text { nocturna } \\
6-8 \text { horas. }\end{array}$ & $\begin{array}{l}\text { La calidad del sueño } \\
\text { en los dos grupos fue } \\
\text { estadísticamente idéntica }\end{array}$ & - & 3 \\
\hline $\begin{array}{l}\text { Sudo, A. } 1996 \\
\text { (38) }\end{array}$ & Transversal & 75 & $93-100$ & $\begin{array}{l}\text { Rendimiento, fatiga } \\
(*) \text { (somnolencia, } \\
\text { embotamiento, } \\
\text { cansancio, } \\
\text { dificultad } \\
\text { de concentración) }\end{array}$ & $\begin{array}{l}\text { Todos los trabajadores } \\
\text { refieren que los EPIs son } \\
\text { útiles frente al ruido y } \\
\text { mejoran las condiciones } \\
\text { de trabajo }\end{array}$ & - & 3 \\
\hline $\begin{array}{c}\text { Kjellberg, A. } \\
1998 \text { (48) }\end{array}$ & Transversal & 24 & $>80-85$ & $\begin{array}{l}\text { Psicosomaticos: } \\
\text { Cefaleas fatiga, } \\
\text { tiempo de reacción, } \\
\text { rendimiento }\end{array}$ & $\begin{array}{l}\text { La aparición de fatiga y } \\
\text { cefalea es más frecuente } \\
\text { en el grupo de mayor } \\
\text { exposición. La } \\
\text { fatiga }>80 \mathrm{dBA} \text {, OR } \\
1.19(1.08-1.30) \text { y mas } \\
\text { cefalea en el grupo entre } \\
60-80 \mathrm{dBA} \text { y }>80 \mathrm{dBA} \text { : } \\
\text { OR } 1.12(1.05-1.19) \text { y OR } \\
1.14(1.02-1.26) \\
\text { respectivamente. El } \\
\text { tiempo de respuesta a la } \\
\text { tarea se relaciona con el } \\
\text { cansancio. El tiempo de } \\
\text { reacción fue en aumento } \\
\text { a lo largo de la semana y } \\
\text { al final de del día }\end{array}$ & - & 3 \\
\hline
\end{tabular}




\begin{tabular}{|c|c|c|c|c|c|c|c|}
\hline $\begin{array}{c}\text { PRIMER } \\
\text { AUTOR } \\
\text { AÑN/ (REF) }\end{array}$ & $\begin{array}{l}\text { TIPO DE } \\
\text { DISEÑO }\end{array}$ & $\mathbf{N}$ & $\begin{array}{l}\text { UMBRAL } \\
\text { DE } \\
\text { RUIDO } \\
\text { dBA }\end{array}$ & $\begin{array}{l}\text { VARIABLE } \\
\text { RESULTADO }\end{array}$ & RESULTADOS & F.C(1) & $\begin{array}{l}\text { N.E. } \\
(2)\end{array}$ \\
\hline $\begin{array}{l}\text { Gomes LM, } \\
1999 \text { (49) }\end{array}$ & Transversal & 40 & $<=90$ & $\begin{array}{l}\text { Función cognitiva, } \\
\text { y de memoria. }\end{array}$ & $\begin{array}{l}\text { En la Escala de Wrestler: } \\
\text { solo la subescala de } \\
\text { memoria inmediata } \\
\text { mostró alguna diferencia } \\
\text { significativa entre } \\
\text { expuestos y no expuestos } \\
\text { (p=0.02).El cociente de } \\
\text { memoria obtuvo } \\
\text { diferencias significativas } \\
\text { entre ambos grupos, con } \\
\text { peores resultados en el } \\
\text { grupo expuesto(p=0,002). } \\
\text { Estos hallazgos sugieren } \\
\text { que la exposición } \\
\text { prolongada a ruido de } \\
\text { baja frecuencia podría } \\
\text { contribuir al deterioro } \\
\text { cognitivo. }\end{array}$ & - & 3 \\
\hline $\begin{array}{l}\text { Sailer U, } 2000 \\
\text { (50) }\end{array}$ & Validación & 116 & 55 & $\begin{array}{l}\text { Malestar en lugares } \\
\text { de oficinas; } \\
\text { validación de } \\
\text { cuestionario SENO }\end{array}$ & $\begin{array}{l}\text { SENO: media } 2.67 \mathrm{SD} \\
0.54 ; \mathrm{n}=114 \\
\text { Lr media 49dBA } \\
(36,5-57,9 \mathrm{dBA} ; \mathrm{n}=91) \\
\text { Correlación }=\mathrm{r}=0.627, \mathrm{~N} \\
=111, \mathrm{p}<0,001 \text { dos } \\
\text { ramas; } \\
\text { No hubo correlación } \\
\text { entre } \mathrm{Lr} \text { y SENO: } \mathrm{r}=0,002 \text {, } \\
\mathrm{n}=89 \text { ns dos ramas }\end{array}$ & - & 3 \\
\hline $\begin{array}{l}\text { Chacín- } \\
\text { Almarza, B. } \\
2002 \text { (51) }\end{array}$ & Transversal & $N=163$ & $87,9-100$ & $\begin{array}{l}\text { Estres } \\
\text { organizacional. } \\
\text { Cuestionario de } \\
\text { ILO/WHO. }\end{array}$ & $\begin{array}{l}\text { No se vio asociación } \\
\text { entre Leq y estrés }\end{array}$ & & 3 \\
\hline $\begin{array}{l}\text { Mahnaz } \\
\text { Saremi } \\
2008(52)\end{array}$ & Transversal & 254 & $>80-85$ & $\begin{array}{l}\text { Rendimiento, } \\
\text { fatiga secundaria al } \\
\text { trabajo. }\end{array}$ & $\begin{array}{l}\text { La exposición a ruido } \\
\text { tiene un efecto } \\
\text { significativo en la fatiga. } \\
\text { ANOVA } \mathrm{F}(2,233)=9,6 \\
\mathrm{p}=0.0001 . \text { Hay interacción } \\
\text { entre la edad y la } \\
\text { exposición a ruido } \\
(\mathrm{F}(2,233)=5,7 \mathrm{p}=.003 \text { (los } \\
\text { mayores tienen mas } \\
\text { fatiga independiente del } \\
\text { nivel de ruido y en los } \\
\text { jóvenes si depende del } \\
\text { nivel) }\end{array}$ & - & 3 \\
\hline $\begin{array}{l}\text { Chiovenda, P } \\
2007 \text { (53) }\end{array}$ & Transversal & 39 & 92 & $\begin{array}{l}\text { Se evaluaron } \\
\text { las funciones } \\
\text { neuropsicológicas } \\
\text { (capacidad de } \\
\text { atención, } \\
\text { memoria de } \\
\text { trabajo o a corto } \\
\text { plazo y capacidad } \\
\text { de razonamiento } \\
\text { lógico) }\end{array}$ & $\begin{array}{l}\text { No se encontraron } \\
\text { diferencias significativas } \\
\text { en los perfiles cognitivos } \\
\text { y emocionales. }\end{array}$ & + & 3 \\
\hline
\end{tabular}

(1) Control de posibles factores de confusión

(2) Nivel de Evidencia

(*)Articulo analizado dos veces por estudiar distintos efectos

17 de los trabajos analizados estudian el efecto de la exposición a ruido y su relación con distintos efectos de naturaleza neuro-psicológica. Con respecto al tipo de diseño, 8 estudios se corresponden con un diseño experimental, 8 estudios son observacionales (1 estudio de cohorte y 7 estudios transversales) y 1 estudio es de validación.

Estos estudios analizan un espectro muy amplio de efectos dentro de los cuales predomina el estudio de efectos relacionados con rendimiento y fatiga (8 artículos), aspectos cognitivos y memoria (5 artículos), estrés y trastornos del humor (4 artículos), 
trastornos del sueño ( 4 estudios), percepción del bienestar ( 1 artículo) y un estudio de validación.

Los umbrales de ruido analizados para estos tipos de efectos oscilan entre 40 y 100 dBA según se trate del estudio de Aspectos cognitivos y memoria (50-92dBA), rendimiento y fatiga (40-100dBA), estrés y trastornos del humor (60-100dBA), trastornos del sueño (75-85dBA) o bienestar (40-55 dBA).

Se observa un acuerdo en los resultados de los estudios tanto experimentales como trasversales que analizan la influencia del ruido en la aparición de la fatiga y disminución del rendimiento. El nivel de evidencia alcanzado para esta asociación es de 1+.

En lo que se refiere a los estudios que analizan efectos en las funciones cognitivas y memoria, se observa un acuerdo en los resultados de los dos estudios de diseño experimental; el primero llevado a cabo por Murthy en 1995 (40), que analiza la asociación entre entorno ruidoso, eficiencia mental y memoria a corto plazo y el segundo, realizado por Pawlaczyk en 2005 (45), que asocia exposición a ruido con alteraciones en la concentración y atención.

Gomes L.M en 1999 (49) en un estudio de diseño trasversal también encuentra una asociación entre exposición a ruido y alteraciones en test de memoria inmediata.

En contrapartida, Chiovenda (53) en un estudio de diseño trasversal sobre capacidad de atención, memoria y capacidad de razonamiento en policías de tráfico no evidencia diferencias significativas para estas funciones, entre el grupo de policías de tráfico y el grupo control.

Con respecto a la influencia de la exposición profesional a ruido y calidad de sueño, los estudios más potentes desde el punto de vista de la evidencia, corresponden a los realizados por el grupo de Gitanjali en 2003, uno de diseño experimental y otro de cohortes.

En el primero (36), llevado a cavo sobre una muestra de 10 trabajadores con monitorización del sueño mediante polisomnografía una noche previa a la exposición ( $>$ de $87 \mathrm{dBA}$ ) y la noche posterior a dicha exposición, no se encuentran diferencias en lo que se refiere a la percepción subjetiva de la calidad del sueño pero sí sobre la arquitectura del sueño, tanto en la duración media de la fase REM como en el tiempo de latencia en su inicio y tiempo de duración de la fase SWS ( $\mathrm{p}>0,0001)$.

En el segundo estudio de cohortes (46) donde se monitoriza el sueño mediante polisomnografía a tres grupos de trabajadores expuestos a más de $75 \mathrm{dBa}$ ruido durante diferentes tiempos de exposición, los resultados revelan una fuerte asociación entre exposición a ruido y baja eficiencia del sueño $(\mathrm{RR}=2,49$; IC 1.12 -5.57). Los cambios más importantes se registraron en el grupo con menor tiempo de exposición (1-2 años) concluyendo que el riesgo de pérdida de calidad del sueño es seguido de una adaptación.

Por otro lado, los resultados de un estudio transversal llevado a cabo por Rios en 2005 (47), en 20 trabajadores expuestos a ruido (exposición equivalente de 85 dB(A) / 40 horas semanales) con un grupo control de 20 trabajadores sin exposición relevante a ruido, ambos con monitorización del sueño mediante polisomnografía, no revelan diferencias ni en la calidad y cantidad de sueño.

Los artículos estudiados orientan la evidencia científica (1+) hacia la influencia de la exposición laboral al ruido y la calidad de sueño, si bien parece ser que ésta influencia negativa no se sostiene en el tiempo (2++).

La no asociación entre exposición a ruido y trastornos de sueño presenta una nivel bajo de evidencia, basado en un estudio de diseño transversal (nivel de evidencia 3).

Con respecto a la asociación entre exposición a ruido, estrés y/o alteraciones del humor tales como irritabilidad, ansiedad, etc los resultados de los estudios experimentales coinciden en revelar una asociación positiva. Por un lado, Melamed en 1996 (41) pone de manifiesto que la exposición laboral al ruido se asocia a un estado de irritabilidad finalizada la jornada laboral, con mejoras significativas al reducir el nivel de exposición 
( $\mathrm{p}<0,01)$ y evidencia la contribución de la exposición ruido en la aparición del estrés laboral.; Por otro lado Ising, en 2004 (37), llega a conclusiones similares, evidenciando asociación entre exposición laboral al ruido, estrés y tensión mental.

Por el contrario, un estudio de corte trasnversal realizado Chazín Almarza en 2002 (51), no encuentra asociación entre exposición a ruido y estrés medido a través del cuestionario de estrés organizacional de ILO/WHO.

Finalmente, la influencia del ruido en el entorno laboral sobre la percepción de bienestar, es analizada por Persson (43) en un estudio de diseño experimental con niveles de exposición habituales en entornos de oficinas resultando una relación entre ruido de baja frecuencia y percepción subjetiva molesta.

Efectos en la salud reproductiva por exposición profesional a ruido (tabla 7).

Tabla 7. Publicaciones analizadas sobre exposición laboral a ruido y salud reproductiva

\begin{tabular}{|c|c|c|c|c|c|c|c|}
\hline $\begin{array}{c}\text { PRIMER } \\
\text { AUTOR } \\
\text { AÑN/ (REF) }\end{array}$ & $\begin{array}{l}\text { TIPO DE } \\
\text { DISEÑO }\end{array}$ & $\mathbf{N}$ & $\begin{array}{c}\text { UMBRAL } \\
\text { DE RUIDO } \\
\text { dBA }\end{array}$ & $\begin{array}{l}\text { VARIABLE } \\
\text { RESULTADO }\end{array}$ & RESULTADOS & F.C. (1) & $\begin{array}{l}\text { N.E. } \\
\text { (2) }\end{array}$ \\
\hline $\begin{array}{l}\text { Wu, TN, } \\
1996(54)\end{array}$ & $\begin{array}{l}\text { Cohorte } \\
\text { prospectiva }\end{array}$ & $\begin{array}{l}200 \\
\text { embarazada. }\end{array}$ & $\begin{array}{l}\text { Leq/24h: } \\
\text { 52-86.8dBA }\end{array}$ & Bajo peso & $\begin{array}{l}\text { No encontraron } \\
\text { asociación } \\
\text { estadística entre } \\
\text { exposición a ruido } \\
\text { y bajo peso }\end{array}$ & + & $2++$ \\
\hline $\begin{array}{l}\text { Luke B } 1995 \\
\text { (55) }\end{array}$ & Casos Control & $\begin{array}{l}\text { Casos: } 210 \\
\text { prematuros de } \\
<37 \text { semanas }\end{array}$ & $\begin{array}{l}\text { "Ruido } \\
\text { constante, alto } \\
\text { y mantenido" } \\
\text { evaluación por } \\
\text { cuestionario }\end{array}$ & Prematuridad & $\begin{array}{l}\text { Asociación entre } \\
\text { ruido y parto } \\
\text { prematuro de } \\
<37 \text { semanas } \\
(\mathrm{p}<0.005)\end{array}$ & + & $2++$ \\
\hline $\begin{array}{l}\text { Savitz, DA, } \\
1996 .(56)\end{array}$ & Casos Control & $\begin{array}{l}216 \text { abortos } \\
315 \\
\text { prematuros }\end{array}$ & $\begin{array}{l}\text { cuestionario de } \\
\text { datos laborales, } \\
\text { de exposición, } \\
\text { y ambientales }\end{array}$ & Prematuridad & $\begin{array}{l}\text { No asociaciones } \\
\text { significativas entre } \\
\text { exposiciones } \\
\text { específicas y efectos } \\
\text { adversos } \\
\text { del parto }\end{array}$ & + & $2++$ \\
\hline $\begin{array}{l}\text { Saurel- } \\
\text { Cubizolles, } \\
\text { MJ 2004(57) }\end{array}$ & Casos Control & $\begin{array}{l}\text { Casos } 2369 \\
\text { pretérminos }\end{array}$ & $\begin{array}{l}\text { Encuesta } \\
\text { laboral }\end{array}$ & Prematuridad & $\begin{array}{l}\text { No relación con } \\
\text { ruido laboral }\end{array}$ & $\begin{array}{l}\text { (-) sesgo } \\
\text { informacion }\end{array}$ & $2-$ \\
\hline $\begin{array}{l}\text { Rocha,EB } \\
2007 \text { (58) }\end{array}$ & Transversal & 35 casos & $\begin{array}{l}\text { Leqd } 8 \mathrm{~h} \\
>80-<90 \mathrm{bBA}\end{array}$ & $\begin{array}{l}\text { Perdida } \\
\text { auditiva } \\
\text { en neonatos }\end{array}$ & $\begin{array}{l}\text { No asociación } \\
\text { estadística entre } \\
\text { ruido y pérdida } \\
\text { auditiva ni con las } \\
\text { medidas de } \\
\text { distorsiones del } \\
\text { oto-emisiones } \\
\text { acústicas en neonatos } \\
\text { entre el grupo } \\
\text { control y el de } \\
\text { estudio. No } \\
\text { diferencias de } \\
\text { lateralidad o genero }\end{array}$ & + & 3 \\
\hline $\begin{array}{l}\text { Spinelli A, } \\
1997 \text { (59) }\end{array}$ & Transversal & 622 & $\begin{array}{l}\text { Ruido } \\
\text { percibido }\end{array}$ & Subfertilidad & No asociación & + & 3 \\
\hline
\end{tabular}

(1) Control de posibles factores de confusión

(2) Nivel de Evidencia

(*)Articulo analizado dos veces por estudiar distintos efectos 
La influencia de la exposición laboral a ruido sobre la salud reproductiva es evaluada en un estudio de cohorte, tres estudios casos-control y dos estudios transversales donde se analiza la Prematuridad ( 3 artículos), el bajo peso al nacer ( 1 artículo), la subfertilidad (1 artículo) y la pérdida auditiva en neonatos (1 artículo).

Luke en 1995 (55) analiza mediante un diseño de casos y controles la incidencia de varios factores laborales (entre ellos el ruido), sobre 210 casos de partos prematuros y 1.260 partos a término en una población de enfermeras. Los resultados evidencian que la exposición a ruido, controlando los factores de confusión, se asocia significativamente con el parto pre-término $(\mathrm{p}<0,005)$.

Con igual diseño de casos-control, Savitz en 1996 (56) estudia el efecto de múltiples exposicones, entre ellas, la exposición profesional a ruido en 216 casos de aborto y 315 casos de parto pretérmino en una población de trabajadoras del sector textil. La exposición profesional a ruido es evaluada de forma subjetiva mediante opinión de expertos en base a inferencia por el tipo de trabajo y contenido de la tarea y por opinión subjetiva del propio trabajador. Los resultados obtenidos muestran valores de OR no significativos para la exposición a ruido y aborto (ORajustado 0,5-1,7), y para el parto -pretérmino (ORajustado 0,4-1,3).

Los resultados del estudio Europop (57), con un diseño de casos-control, llevado a cabo en una población de mujeres europeas trabajadoras que al menos han mantenido su actividad laboral durante los tres primeros meses de embarazo con 2.369 casos de parto prematuro y 4.098 controles de embarazo a término, no encuentra asociación entre exposición profesional a ruido, evaluada de forma subjetiva y parto- pretérmino (ORajustado 0,9- 1,1)

La influencia de la exposición profesional al ruido durante el embarazo y el peso del feto al nacer es estudiada por Trong- Neng Wu en 1996 (54) a través del seguimiento de 200 embarazas hasta el momento del parto. Los resultados no revelan asociación entre exposición profesional a ruido, evaluada mediante dosimetría a las 15, 25 y 30-40 semanas de embarazo y bajo peso al nacer. La exposición media a ruido se situó en un rango entre 52,4 a $86,8 \mathrm{~dB}(\mathrm{~A}) \mathrm{Leq}_{24}$

Spinelli (59) en 1997 mediante un diseño transversal analiza el tiempo de espera para el embarazo y la exposición profesional a diferentes riesgos, incluido la exposición a ruido, de 622 mujeres con partos a término. Los resultados no evidencian relación entre exposición laboral a ruido y tiempo de espera para el embarazo. La razón de tiempo de espera entre trabajadoras expuestas a ruido y no expuestas fue de 1,08 $(0,81-1,43)$.

Rocha (58) en 2007 mediante un diseño transversal estudia las lesiones audiológicas de 80 niños entre 0 y 6 meses de edad, 35 niños procedentes de madres trabajadoras expuestas durante su embarazo a ruido (rango de 80- 90 dBSPL) y 45 niños de madres no expuestas. Los resultados de Rocha no muestran diferencias (mediante t-test) en los parámetros oto-acústicos analizados entre los dos grupos de estudio, no evidenciándose una influencia de la exposición profesional durante el embarazo a los niveles de ruido analizados y el déficit auditivo de los hijos.

Efectos de exposición profesional a ruido en la accidentabilidad profesional (tabla 8). 
Tabla 8. Publicaciones analizadas sobre exposición laboral a ruido y accidentes de trabajo

\begin{tabular}{|c|c|c|c|c|c|c|c|}
\hline $\begin{array}{c}\text { PRIMER } \\
\text { AUTOR } \\
\text { AÑNO/ (REF) }\end{array}$ & $\begin{array}{l}\text { TIPO DE } \\
\text { DISEÑO }\end{array}$ & $\mathbf{N}$ & $\begin{array}{c}\text { UMBRAL } \\
\text { DE RUIDO } \\
\text { dBA }\end{array}$ & $\begin{array}{c}\text { VARIABLE } \\
\text { RESULTADO }\end{array}$ & RESULTADOS & $\begin{array}{l}\text { F.C. } \\
\text { (1) }\end{array}$ & $\begin{array}{l}\text { N.E. } \\
\text { (2) }\end{array}$ \\
\hline $\begin{array}{l}\text { Barreto, SMl } \\
1997 \text { (60) }\end{array}$ & Caso-control & $\begin{array}{l}37 \\
\text { casos }\end{array}$ & $\begin{array}{l}\text { Leq/d8h: } \\
\text { bajo ( } 85-89) \\
\text { moderado } \\
(90-94) y \\
\text { alto } \\
(>=95 \text { dBA })\end{array}$ & $\begin{array}{l}\text { Riesgo } \\
\text { de sufrir } \\
\text { accidentes } \\
\text { de trabajo } \\
\text { mortales }\end{array}$ & $\begin{array}{l}\text { El riesgo de accidentes } \\
\text { mortales fue de OR aj. } \\
\text { ruido moderado } 5,72 \\
(1,63-20,11) \text { OR aj. } \\
\text { ruido alto) } 4,23 \\
(1.19-15.32)\end{array}$ & $(+)$ & $2++$ \\
\hline $\begin{array}{l}\text { Cordeiro R, } \\
2005 \text { (61) }\end{array}$ & Caso-control & $\begin{array}{l}94 \\
\text { casos } \\
(3: 1)\end{array}$ & $\begin{array}{l}\text { Valoración } \\
\text { subjetiva } \\
\text { por } \\
\text { preguntas }\end{array}$ & $\begin{array}{l}\text { Accidentes } \\
\text { de trabajo }\end{array}$ & $\begin{array}{l}\text { Para la respuesta } \\
\text { "siempre expuesto a } \\
\text { alto nivel de ruido: } \mathrm{RR} \\
=5(95 \% 2.8-8.7 \\
\text { p<0,001) y para } \\
\text { "algunas veces } \\
\text { expuesto a alto nivel } \\
\text { de ruido" el } \mathrm{RR}=3 \\
(1.8-7.4 \text { p }<0,0003) .\end{array}$ & $(+)$ & $2++$ \\
\hline $\begin{array}{l}\text { Dias, A } \\
2007(62)\end{array}$ & Caso-control & $\begin{array}{l}600 \\
\text { casos }\end{array}$ & $\begin{array}{l}\text { preguntas } \\
\text { con } 4 \\
\text { niveles }\end{array}$ & $\begin{array}{l}\text { Accidentes } \\
\text { de trabajo }\end{array}$ & $\begin{array}{l}\text { FA }=0,30(0,23-0,36) \\
\text { OR para intensidad } \\
\text { media de ruido } 1,6(p \\
=0,003 ; 1,17-2,26) \\
\text { OR para alta } \\
\text { intensidad } 2,29 \\
(p>0,0001 ; 1,51-3,47)\end{array}$ & $(+)$ & $2++$ \\
\hline $\begin{array}{l}\text { Melamed, S } \\
2004(63)\end{array}$ & Transversal & $\begin{array}{l}4084 \\
\text { varones } \\
\text { y } 1643 \\
\text { mujeres }\end{array}$ & $>=80 \mathrm{dBA}$ & $\begin{array}{l}\text { Accidentes } \\
\text { de trabajo y } \\
\text { ansiedad }\end{array}$ & $\begin{array}{l}\text { El Riesgo más alto de } \\
\text { accidente se produce } \\
\text { en mujeres con alto } \\
\text { nivel de exposición a } \\
\text { ruido y tareas } \\
\text { complejas }(\mathrm{OR}=2,72 \text {, } \\
\text { IC } 1,06-6,97 ; \mathrm{p}= \\
0,036) \text {. } \\
\text { OR varones }=1,3 \\
(1-1,69 ; \mathrm{p}=0,050) ;\end{array}$ & $(+)$ & 3 \\
\hline
\end{tabular}

(1) Control de posibles factores de confusión

(2) Nivel de Evidencia

(*)Articulo analizado dos veces por estudiar distintos efectos

La influencia de la exposición laboral a ruido sobre la accidentalidad se analiza en cuatro estudios con diseño observacional: tres estudios casos- control y un estudio transversal.

Los resultados de los tres estudios casos-control son coincidentes y evidencian un incremento del riesgo para el accidente de trabajo en trabajadores expuestos a niveles moderados o intensos de exposición a ruido. Los valores de OR que se obtienen oscilan entre1,6, según Dias A (62) y 5,72 según Barreto (60).

Melamed (63) en un diseño transversal obtiene resultados divergentes entre mujeres y varones evidenciando sólo valores de riesgo para mujeres que desarrollan tareas de alta complejidad.

\section{DISCUSIÓN}

Los resultados de esta revisión son coincidentes con los resultados de las revisiones realizadas Smith AP y cols (5) y Butler MP y cols (23), en relación con la influencia de la exposición a ruido y secreción de cortisol y noradreanalina.

Desde el punto de vista de la práctica de la vigilancia de la salud de los trabajadores, la determinación de cortisol y/o noradrenalina en diferentes especímenes son marcadores útiles para detectar precozmente cambios secundarios a estrés fisiológico desencadenante de enfermedades en el sistema cardiovascular, gastrointestinal y respiratorio entre otros. 
Butler y cols intentan confirmar los resultados de la revisión de Smith y Broadbent sobre la percepción de malestar y exposición a ruido. Su revisión incluye estudios que analizan como efecto de la exposición al ruido alteraciones en el área psicosocial (64-66)

La revisión de Butler concluye que el ruido aumenta el malestar, pero observan muy pocos efectos directos del ruido sobre alteraciones psicopatológicas en el individuo, por lo que el grado de malestar en muchas ocasiones puede estar reflejando las características psicológicas del individuo (67).

El malestar por ruido puede ser un predictor de algunos efectos negativos, estando documentado a niveles de exposición a ruido de $51 \mathrm{~dB}(\mathrm{~A})$, muy por debajo de los niveles umbrales de exposición laboral (68). En numerosos diseños no queda reflejado el control de factores de confusión (69).

En el caso de los efectos englobados en nuestra revisión como psicosociales, obtenemos resultados variables:

Así la relación entre exposición profesional al ruido y malestar encuentra un alto nivel de evidencia, hecho que puede considerarse como una percepción que afecta a la calidad de vida más que una alteración de la salud propiamente dicha.

El ruido parece que influye en el sueño de varias formas; prolongando el tiempo de inducción, despertar precoz, disminución del sueño profundo e incrementando el superficial (70-73). La mayoría de los estudios que analizan los trastornos del sueño se han realizados con exposiciones de carácter ambiental (12). En nuestra revisión la evidencia encontrada habla a favor de una alteración en la calidad del sueño en trabajadores expuestos a ruido.

En la revisión de Butler y cols sólo se analizan dos estudios $(74 ; 75)$ que relacionan el ruido con el nivel de fatiga pero ambos estudios tienen la exposición a vibraciones como factor de confusión.

Butler y col (23) analizan la asociación entre ruido y rendimiento en poblaciones no laborales por lo que sus conclusiones son difícilmente extrapolables a entornos de trabajo (76).

Además bajo la variable rendimiento se agrupan diversos efectos (77) que dificultan también su comparación.

Algunos estudios, sobre todo de diseño experimental, mostraron que las alteraciones en el rendimiento pueden aparecer por debajo de niveles de exposición, entre 85 y 90dBA, si bien estudios también experimentales evidencian que el ruido moderado puede mejorar la actividad laboral (78).

La revisión de estudios realizados en población laboral concluyen que el ruido puede tener un efecto sobre el rendimiento $(79 ; 80)$.

En nuestra revisión encontramos un alto nivel de evidencia (1+) y una coincidencia en la dirección de los resultados de la repercusión de la exposición a ruido sobre la disminución del rendimiento, repercusión sobre la productividad y sobre la fatiga.

Hasta la revisión de Butler y cols (23) se había sugerido que la exposición a ruido en entornos laborales durante el embarazo se podía asociar con anormalidades de la función reproductiva y defectos congénitos $(15 ; 81 ; 82)$. Butler y cols, analizan 7 artículos originales sobre el efecto del ruido y la salud reproductiva (un estudio experimental, tres estudios transversales, dos estudios de cohorte y un estudio casos control). De acuerdo a su revisión, existen resultados contradictorios en lo que se refiere a la influencia de la exposición profesional al ruido y bajo peso al nacer, Hartikainen (83) en un diseño de cohorte encuentra resultados significativos, mientras que Henriksen (84), en un diseño también de cohorte encuentra resultados negativos. 
Con respecto a la influencia de la exposición materna al ruido sobre el daño auditivo en el feto, Butler identifica un único estudio de diseño transversal cuyo resultado habla de una asociación positiva (85).

Los estudios revisados sobre parto pre-término presentan resultados coincidentes en la no asociación entre exposición materna a ruido durante el embarazo y prematuridad.

Los resultados de nuestra revisión, al igual que los de Bulter no son concluyentes en lo que se refiere a la salud reproductiva y respecto a la prematuridad los estudios que hemos revisado, presentan evidencias contradictorias.

Los estudios analizados sobre subfertilidad, bajo peso al nacer y lesiones auditivas en neonatos de madres laboralmente expuestas a ruido evidencian una no asociación entre exposición y salud reproductiva.

En cualquier caso estos resultados no son concluyentes bien porque la evidencia se basa en diseños débiles o bien por la escasa producción científica que aborda el estudio de estos efectos. Por otra parte la mayor parte de estudios evalúan la exposición materna a ruido de forma subjetiva.

Con respecto a la influencia de la exposición profesional al ruido sobre la accidentalidad laboral nuestros resultados confirman las evidencias encontradas por Smith y Broadbent. Existe un alto nivel de evidencia y coincidencia en los resultados de los estudios que hemos analizado, en lo que se refiere a la exposición profesional al ruido y accidentes de trabajo. No obstante, si bien estos estudios tienen un diseño epidemiológico sólido (casos control), los niveles de exposición analizados era superiores a $80 \mathrm{~dB}(\mathrm{~A})$.

\section{CONCLUSIONES}

En total se han analizado 35 estudios sobre exposición profesional a ruido y efectos extra-auditivos no cardiovasculares: 14 estudios experimentales, 2 estudios de cohortes, 6 estudios casos control, 12 estudios transversales y 1 estudio de validación.

Los efectos analizados incluyeron: concentración y secreción de cortisol, catecolaminas, iones ( $\mathrm{Ca}, \mathrm{P}, \mathrm{Mn}$ ), efectos neuropsicológicos (memoria, estrés, funciones cognitivas, sueño, irritabilidad, fatiga, rendimiento y alteraciones del humor), percepción del malestar, efectos sobre la salud reproductiva (prematuridad, bajo peso, subfertilidad, daños audiológicos fetales) y accidentes de trabajo.

En relación al nivel de evidencia de la asociación entre exposición profesional a ruido y los distintos efectos analizados, encontramos:

1. Exposición profesional a ruido y sus posibles efectos sobre los niveles de cortisol, catecolaminas y otros efectos bioquímicos.

- Las evidencias existentes sobre la relación entre exposición profesional a ruido y este grupo de efectos se basan, fundamentalmente, en trabajos con diseño experimental.

- Existe un alto nivel de evidencia (1+) sobre la influencia de la exposición profesional al ruido en el incremento de la secreción de cortisol y noradrenalina, así como sobre el incremento de CgA en saliva. No existe divergencia de resultados en los estudios analizados.

- La exposición profesional a ruido incrementa también los niveles séricos de Ca y $\mathrm{Mg}$ (nivel de evidencia 1+)

- Con respecto a otras catecolaminas los estudios muestran, con un nivel de evidencia 1+, que la exposición laboral al ruido no tiene efecto sobre su secreción.

- En la interpretación de estos niveles de evidencia hay que considerar que los umbrales de exposición estudiados se sitúan en el rango de 60 a 108 dB(A). 
2. Exposición profesional a ruido y sus efectos neuropsicológicos.

- Este grupo está integrado por un conjunto de efectos relacionados con la aparición de fatiga y rendimiento, funciones cognitivas y memoria, calidad del sueño, estrés y alteraciones del humor.

- Los resultados de los ocho estudios analizados, tanto de diseño trasversal como experimental son coincidentes en mostrar una relación entre exposición a ruido con la aparición de fatiga y disminución en el rendimiento, con un nivel de evidencia $1+$.

- Los umbrales de ruido analizados en estos estudios oscilaron entre 40 y 100 dB(A).

- La evidencia de la relación entre exposición a ruido sobre las funciones cognitivas y memoria, alcanza un nivel 1+, evidencia basada en dos estudios de diseño experimental, siendo coincidentes en sus resultados. Sin embargo los estudios de diseño transversal presentan resultados contradictorios sobre la exposición a ruido y los perfiles de cognitivos.

- Los umbrales de ruido analizados para lo estudios revisados sobre fatiga y disminución de rendimiento oscilaron entre 50 y $92 \mathrm{~dB}(\mathrm{~A})$.

- La relación entre exposición profesional a ruido y sus efectos sobre la calidad de sueño alcanza un nivel de evidencia 1+ basada en un estudio de diseño experimental, coincidente con los resultados de un estudio de cohorte. Este último estudio pone de manifiesto una adaptación en el tiempo, reduciéndose el efecto de la exposición al ruido sobre la calidad del sueño (nivel de evidencia $2++$ ).

- Los umbrales de exposición de los estudios analizados se encontraban entre 75 y $85 \mathrm{~dB}(\mathrm{~A})$.

- En lo que se refiere al estrés e irritabilidad, existe coincidencia en los estudios experimentales, evidenciando una influencia de la exposición profesional a ruido con el incremento del estrés e irritabilidad (nivel de evidencia 1+). Sólo un estudio transversal (nivel de evidencia 3) presenta resultados no coincidentes con los estudios experimentales, no encontrando relación entre exposición a ruido y estrés.

- Los umbrales de exposición analizados en los estudios revisados sobre estrés e irritabilidad oscilaron entre 60 y $100 \mathrm{~dB}(\mathrm{~A})$.

- La percepción de malestar es analizada para valores de exposición de $40 \mathrm{~dB}(\mathrm{~A})$ mediante un diseño experimental. Sus resultados revelan un nivel de evidencia 1+ para la asociación entre exposición a ruido y percepción de malestar.

3. Exposición profesional a ruido y efectos sobre la salud reproductiva.

- Los resultados en relación con el efecto del ruido sobre la salud reproductiva no son concluyentes.

- En relación con la exposición a ruido, evaluada de forma subjetiva, y parto pretérmino, los tres estudios de diseño casos-control analizados presentan resultados contradictorios.

- Existe evidencia de nivel $2++$, basada en un estudio de cohorte, de la no influencia de la exposición profesional a ruido sobre el peso al nacer.

- Existe una evidencia débil (nivel 3) sobre la no influencia de la exposición profesional al ruido, subfertilidad y daño audiológico en neonatos.

- En todos los estudios analizados, salvo para la influencia sobre el bajo peso al nacer, la evaluación de la exposición a ruido se realizó mediante valoración subjetiva de la trabajadora.

4. Exposición profesional a ruido y accidente de trabajo.

- Los estudios analizados son coincidentes en sus resultados, el nivel de evidencia de la relación entre accidentes de trabajo y exposición profesional a ruido es de $2++$, basada en diseños casos- control.

- Los umbrales de exposición a ruido fueron superiores a $80 \mathrm{~dB}(\mathrm{~A})$ en todos $\operatorname{los}$ estudios. 


\section{BIBLIOGRAFIA}

1. Parent-Thirion A, Fernández Macías E, Hurley J, Vermeylen G. Fourth European Working Conditions Survey. 22-2-2007. European Foundation for the Improvement of Living and Working Conditions.

2. INSHT. VI ENCUESTA NACIONAL DE CONDICIONES DE TRABAJO. 2008. Ministerio de trabajo y asuntos sociales.

3. Ministerio de Trabajo y Asuntos Sociales. REAL DECRETO 286/2006, de 10 de marzo, sobre la protección de la salud y la seguridad de los trabajadores contra los riesgos relacionados con la exposición al ruido. 2006.

4. Instituto sindical de trabajo aysI. Especialidad de higiene industrial del plan de formacion 2006 para empleados de la sanidad publica. 2006. CC.OO, federacion de sanidad y sectores sociosanitarios.

5. Smith AP, Broadbent DE. Non-auditory effects of noise at work:A review of the literature. HSE HSE, editor. 30/1991. 1991.

6. Berglund B LTaSDH. Guidelines for Community Noise. World Health Organization. 1999.

7. Babisch W. The Noise/Stress Concept, Risk Assessment and Research Needs. Noise Health 2002; 4(16):1-11.

8. Spreng M. Possible health effects of noise induced cortisol increase. Noise Health 2000; 2(7):59-64.

9. Alves-Pereira M, Reis Ferreira JM, Joanaz de MJ, Motylewski J, Kotlicka E, Castelo Branco NA. Noise and the respiratory system. Rev Port Pneumol 2003; 9(5):367-379.

10. Kryter K. The effects of noise on man. Academic Pres L, editor. Second edition. 1985.

11. Cortés Barragán, R Maqueda Blasco, J; Ordaz Castillo, E; Asúnsolo del Barco,A; Silva Mato, A; Bermejo García, E; Gamo González; MF:.Revisión sistemática y evidencia sobre exposición profesional a ruido y efectos extraauditivos de naturaleza cardiovascular. Med. segur. trab. [online]. 2009, vol.55, n.215, pp. 28-51. I

12. World Health Organization. Development of Environment and health indicators for European Unions Countries: Results of a Pilot Study. Who working group. 2004. Bonn.

13. Muzat A. Environmental noise, sleep and health. Sleep Med Rev 2007; 11(2):135-42.

14. Leventhall HG. Low frequency noise and annoyance. Noise Health 2004; 6(23):59-72.

15. Knipschild P, Meijer H, Salle H. Aircraft noise and birth weight. Int Arch Occup Environ Health 1981; 48(2):131-136.

16. Peoples-Sheps MD, Siegel E, Suchindran CM, Origasa H, Ware A, Barakat A. Characteristics of maternal employment during pregnancy: effects on low birthweight. Am J Public Health 1991; 81(8):1007-1012.

17. Mamelle N, Laumon B, Lazar P. Prematurity and occupational activity during pregnancy. Am J Epidemiol 1984; 119(3):309-322.

18. Meyer RE, Aldrich TE, Easterly CE. Effects of noise and electromagnetic fields on reproductive outcomes. Environ Health Perspect 1989; 81:193-200.

19. Long JG, Lucey JF, Philip AG. Noise and hypoxemia in the intensive care nursery. Pediatrics 1980; 65(1):143-145.

20. Industrial noise and medical absence and accident record data on exposed workers:The International Congress on Noise as a Public Health Problem. Washington (DC): 1976.

21. Moll van Charante AW, Mulder PG. Perceptual acuity and the risk of industrial accidents. Am J Epidemiol 1990; 131(4):652-663.

22. Murillo IC. [How does noise affect us? In our health, life styles and environs]. Rev Enferm 2007; 30(10):13-20.

23. Butler MP, Graveling RA. NON-AUDITORY EFFECTS OF NOISE AT WORK: A CRITICAL REVIEW OF THE LITERATURE POST 1988. HSE Books, 1999.

24. Scottish Intercollegiate Guidelines Network. SIGN 50: A guideline developer's handbook. http://www.sign. ac.uk. 2008

25. von Elm E, Altman DG, Egger M, Pocock SJ, Gotzsche PC, Vandenbroucke JP. [The Strengthening the Reporting of Observational Studies in Epidemiology (STROBE) statement: guidelines for reporting observational studies]. Rev Esp Salud Publica 2008; 82(3):251-259.

26. Oxman AD, Cook DJ, Guyatt GH. Users' guides to the medical literature. VI. How to use an overview. Evidence-Based Medicine Working Group. JAMA 1994; 272(17):1367-1371.

27. McBride P. Another view on overviews. JAMA 1995; 274(3):217-218.

28. Oxman AD, Sackett DL, Guyatt GH. Users' guides to the medical literature. I. How to get started. The Evidence-Based Medicine Working Group. JAMA 1993; 270(17):2093-2095. 
29. Jaeschke R, Guyatt GH, Sackett DL. Users' guides to the medical literature. III. How to use an article about a diagnostic test. B. What are the results and will they help me in caring for my patients? The EvidenceBased Medicine Working Group. JAMA 1994; 271(9):703-707.

30. Laupacis A, Wells G, Richardson WS, Tugwell P. Users' guides to the medical literature. V. How to use an article about prognosis. Evidence-Based Medicine Working Group. JAMA 1994; 272(3):234-237.

31. Guyatt GH, Sackett DL, Cook DJ. Users' guides to the medical literature. II. How to use an article about therapy or prevention. A. Are the results of the study valid? Evidence-Based Medicine Working Group. JAMA 1993; $270(21): 2598-2601$

32. Melamed S, Bruhis S. The effects of chronic industrial noise exposure on urinary cortisol, fatigue and irritability: a controlled field experiment. J Occup Environ Med 1996; 38(3):252-256.

33. Miki K, Kawamorita K, Araga Y, Musha T, Sudo A. Urinary and salivary stress hormone levels while performing arithmetic calculation in a noisy environment. Ind Health 1998; 36(1):66-69.

34. Mocci F, Canalis P, Tomasi PA, Casu F, Pettinato S. The effect of noise on serum and urinary magnesium and catecholamines in humans. Occup Med (Lond) 2001; 51(1):56-61.

35. Miyakawa M, Matsui T, Kishikawa H, Murayama R, Uchiyama I, Itoh T et al. Salivary chromogranin A as a measure of stress response to noise. Noise Health 2006; 8(32):108-113.

36. Gitanjali B, Ananth R. Effect of acute exposure to loud occupational noise during daytime on the nocturnal sleep architecture, heart rate, and cortisol secretion in healthy volunteers. J Occup Health 2003; 45(3):146-152.

37. Ising H, Michalak R. Stress effects of noise in a field experiment in comparison to reactions to short term noise exposure in the laboratory. Noise Health 2004; 6(24):1-7.

38. Sudo A, Nguyen AL, Jonai H, Matsuda S, Villanueva MB, Sotoyama M et al. Effects of earplugs on catecholamine and cortisol excretion in noise-exposed textile workers. Ind Health 1996; 34(3):279-286.

39. Rojas-Gonzalez L, Martinez-Leal R, Paz-Araviche V, Chacin-Almarza B, Corzo-Alvarez G, Sanabria-Vera C et al. [Serum cortisol levels in pre and post journal labor and non auditory manifestations in noise exposed workers of a brewer industry]. Invest Clin 2004; 45(4):297-307.

40. Murthy VS, Malhotra SK, Bala I, Raghunathan M. Detrimental effects of noise on anaesthetists. Can J Anaesth 1995; 42(7):608-611.

41. Melamed S, Bruhis S. The effects of chronic industrial noise exposure on urinary cortisol, fatigue and irritability: a controlled field experiment. J Occup Environ Med 1996; 38(3):252-256.

42. Miki K, Kawamorita K, Araga Y, Musha T, Sudo A. Urinary and salivary stress hormone levels while performing arithmetic calculation in a noisy environment. Ind Health 1998; 36(1):66-69.

43. Persson WK, Bengtsson J, Kjellberg A, Benton S. Low frequency noise "pollution" interferes with performance. Noise Health 2001; 4(13):33-49.

44. Muzammil M, Khan AA, Hasan F, Hasan SN. Effect of noise on human performance under variable load in a die casting industry--a case study. J Environ Sci Eng 2004; 46(1):49-54.

45. Pawlaczyk-Luszczyniska M, Dudarewicz A, Waszkowska M, Szymczak W, Sliwinska-Kowalska M. The impact of low-frequency noise on human mental performance. Int J Occup Med Environ Health 2005; 18(2):185-198.

46. Gitanjali B, Dhamotharan R. Effect of occupational noise on the nocturnal sleep architecture of healthy subjects. Indian J Physiol Pharmacol 2003; 47(4):415-422.

47. Rios AL, da Silva GA. Sleep quality in noise exposed Brazilian workers. Noise Health 2005; 7(29):1-6.

48. Kjellberg A, Muhr P, Skoldstrom B. Fatigue after work in noise - an epidemiological survey study and three quasi-experimental field studies. Noise Health 1998; 1(1):47-55.

49. Gomes LM, Martinho Pimenta AJ, Castelo Branco NA. Effects of occupational exposure to low frequency noise on cognition. Aviat Space Environ Med 1999; 70(3 Pt 2):A115-A118.

50. Sailer U, Hassenzahl M. Assessing noise annoyance: an improvement-oriented approach. Ergonomics 2000; 43(11):1920-1938.

51. Chacin-Almarza B, Corzo-Alvarez G, Rojas-Gonzalez L, Rodriguez-Chacin E, Corzo-Rios G. [Organizational stress and noise exposure in workers at the packing plant of a brewer factory]. Invest Clin 2002; 43(4):271-289.

52. Saremi M, Rohmer O, Burgmeier A, Bonnefond A, Muzet A, Tassi P. Combined effects of noise and shift work on fatigue as a function of age. Int J Occup Saf Ergon 2008; 14(4):387-394.

53. Chiovenda P, Pasqualetti P, Zappasodi F, Ercolani M, Milazzo D, Tomei G et al. Environmental noise-exposed workers: event-related potentials, neuropsychological and mood assessment. Int J Psychophysiol 2007; 65(3):228-237.

54. Wu TN, Chen LJ, Lai JS, Ko GN, Shen CY, Chang PY. Prospective study of noise exposure during pregnancy on birth weight. Am J Epidemiol 1996; 143(8):792-796. 
55. Luke B, Mamelle N, Keith L, Munoz F, Minogue J, Papiernik E et al. The association between occupational factors and preterm birth: a United States nurses' study. Research Committee of the Association of Women's Health, Obstetric, and Neonatal Nurses. Am J Obstet Gynecol 1995; $173(3$ Pt 1):849-862.

56. Savitz DA, Brett KM, Baird NJ, Tse CK. Male and female employment in the textile industry in relation to miscarriage and preterm delivery. Am J Ind Med 1996; 30(3):307-316.

57. Saurel-Cubizolles MJ, Zeitlin J, Lelong N, Papiernik E, Di Renzo GC, Breart G. Employment, working conditions, and preterm birth: results from the Europop case-control survey. J Epidemiol Community Health 2004; 58(5):395-401.

58. Rocha EB, Frasson dA, Ximenes Filho JA. Study of the hearing in children born from pregnant women exposed to occupational noise: assessment by distortion product otoacoustic emissions. Braz J Otorhinolaryngol 2007; 73(3):359-369.

59. Spinelli A, Figa-Talamanca I, Osborn J. Time to pregnancy and occupation in a group Barreto SM, Swerdlow AJ, Smith PG, Higgins CD. A nested case-control study of fatal work related injuries among Brazilian steel workers. Occup Environ Med 1997; 54(8):599-604.

60. Barreto,S.M.; Swerdlow,A.J.; Smith,P.G.; Higgins,C.D. A nested case-control study of fatal work related injuries among Brazilian steel workers. Occup Environ Med, 54 (8) 599-604.

61. Cordeiro R, Clemente AP, Diniz CS, Dias A. [Occupational noise as a risk factor for work-related injuries]. Rev Saude Publica 2005; 39(3):461-466.

62. Dias A, Cordeiro R. Attributable fraction of work accidents related to occupational noise exposure in a Southeastern city of Brazil. Cad Saude Publica 2007; 23(7):1649-1655.

63. Melamed S, Fried Y, Froom P. The joint effect of noise exposure and job complexity on distress and injury risk among men and women: the cardiovascular occupational risk factors determination in Israel study. $\mathrm{J}$ Occup Environ Med 2004; 46(10):1023-1032.

64. Ivanovich E, Kolarova D, Enev S, Tzenova B, Topalova M. Noise evaluation and estimation of some specific and non-specific health indicators in telephone operators. Rev Environ Health 1994; 10(1):39-46.

65. Topf M. Personality hardiness, occupational stress, and burnout in critical care nurses. Res Nurs Health 1989; 12(3):179-186.

66. Stansfeld SA. Noise, noise sensitivity and psychiatric disorder: epidemiological and psychophysiological studies. Psychol Med 1992; Suppl 22:1-44.

67. Ekehammar B, Dornic S. Weinstein's Noise Sensitivity Scale: reliability and construct validity. Percept Mot Skills 1990; 70(1):129-130.

68. Kjellberg A. Subjective, behavioral and psychophysiological effects of noise. Scand J Work Environ Health 1990; 16 Suppl 1:29-38.

69. Mc Donald N, Ronayne T. Jobs and the environment: the psychological impact of work in noise. Irish Journal of Psycology 1989; 10:39-55.

70. Topf M. Hospital noise pollution: an environmental stress model to guide research and clinical interventions. J Adv Nurs 2000; 31(3):520-528.

71. Carter NL, Hunyor SN, Crawford G, Kelly D, Smith AJ. Environmental noise and sleep--a study of arousals, cardiac arrhythmia and urinary catecholamines. Sleep 1994; 17(4):298-307.

72. Nivison ME, Endresen IM. An analysis of relationships among environmental noise, annoyance and sensitivity to noise, and the consequences for health and sleep. J Behav Med 1993; 16(3):257-276.

73. Libert JP, Bach V, Johnson LC, Ehrhart J, Wittersheim G, Keller D. Relative and combined effects of heat and noise exposure on sleep in humans. Sleep 1991; 14(1):24-31.

74. Effect of noise and vibration on the vigilance of drivers of light vehicles. 91 Nov 21; Brussels: 1991.

75. Kjellberg A, Sköldström B, Anderssson P, Lindberg L. Fatigue effects of noise on aeroplane mechanics. Work and Stress 1996; 10:62-71.

76. Bhattacharya SK, Pradhan CK, Tripathi SR, Kashyap S. Human performance capability in psychomotor tasks at variable difficulty levels and physiological reactions under noise and heat conditions. Ind Health 1991; 29(4):129-138

77. Kjellberg A, Skoldstrom B. Noise annoyance during the performance of different nonauditory tasks. Percept Mot Skills 1991; 73(1):39-49.

78. Britton LA, Delay ER. Effects of noise on a simple visual attentional task. Percept Mot Skills 1989; 68(3 Pt 1):875-878.

79. Rentzsch M. Interactions between different types of industrial noises and work tasks. Environmental international 1990; 16:459-470.

80. Smith A. A review of the effects of noise on human performance. Scand J Psychol 1989; 30(3):185-206. 
81. Ando Y, Hattori H. Effects of intense noise during fetal life upon postnatal adaptability (statistical study of the reactions of babies to aircraft noise). J Acoust Soc Am 1970; 47(4):1128-1130.

82. Ando Y, Hattori H. Effects of noise on human placental lactogen (HPL) levels in maternal plasma. Br J Obstet Gynaecol 1977; 84(2):115-118.

83. Hartikainen AL, Sorri M, Anttonen H, Tuimala R, Laara E. Effect of occupational noise on the course and outcome of pregnancy. Scand J Work Environ Health 1994; 20(6):444-450

84. Henriksen,T.B.; Hedegaard,M.; Secher,N.J. The relation between psychosocial job strain, and preterm delivery and low birthweight for gestational age. Int J Epidemiol, 23 (4); 764-774.

85. Lalande NM, Hetu R, Lambert J. Is occupational noise exposure during pregnancy a risk factor of damage to the auditory system of the fetus? Am J Ind Med 1986; 10(4):427-435.

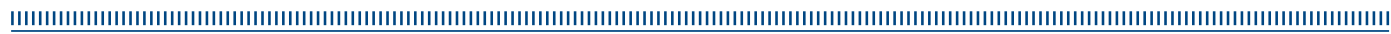

